\title{
Head and Neck Squamous Cell Carcinoma Detection and Surveillance: Advances of Liquid Biomarkers
}

\author{
Paul L. Swiecicki, MD; Julia R. Brennan, BSE; Michelle Mierzwa, MD; \\ Matthew E. Spector, MD $\mathbb{D}$; J. Chad Brenner, PhD
}

\begin{abstract}
Head and neck squamous cell carcinomas are aggressive tumors that often present at advanced stage in difficult-to-biopsy regions of the head and neck. With the rapid move to analyze circulating tumor DNA (ctDNA) to either detect cancer or monitor disease progression and response to therapy, we have designed this article as a primer to understand the recent studies that support a transition to use these circulating biomarkers as a part of routine clinical care. Whereas some technical challenges still need to be overcome, the utility of ctDNA in cancer care is already evident from these early studies. Therefore, it is critical to understand recent advances in this area as well as emerging questions that need to be addressed as these biomarkers move closer to enhancing routine clinical care paradigms.
\end{abstract}

Key Words: ctDNA, HPV, biomarker, HRAS, ccfDNA.

Laryngoscope, 129:1836-1843, 2019

\section{INTRODUCTION}

Head and neck cancer is one of the leading causes of cancer deaths worldwide. ${ }^{1}$ Head and neck squamous cell carcinoma (HNSCC) comprises the vast array of tumors, including those arising from the nose, nasal cavity, paranasal sinuses, oral cavity, pharynx, and larynx. Within the last several decades, the rising incidence of oropharyngeal cancer, particularly in the younger population, has been attributed to high-risk human papillomavirus (HPV) subtypes ${ }^{2}$ as well as potential hereditary genetic factors. ${ }^{3,4}$ The detection of primary HNSCC and recurrence is challenging due to the nature of the anatomy involved. Diagnosis is often delayed and requires clinical examination, imaging, and microscopic tissue analysis, all of which can be significantly hindered by the location of the tumors. There is significant interest in the development of a biomarker surveillance tool that might allow for earlier diagnosis and bypass the need to undergo a surgical procedure. In this review article, we performed a systematic review of the literature, focusing on the development of serum-based biomarkers, particularly circulating tumor DNA (ctDNA), in HNSCC. We included a

From the Department of Internal Medicine, Division of Hematology and Oncology (P.L.S.); the Department of OtolaryngologyHead and Neck Surgery (J.R.B., M.E.S., J.C.B.); the Department of Radiation Oncology (м.м.); the Program in Cellular and Molecular Biology (J.с.в.); the Department of Pharmacology (J.c.в.); and the Comprehensive Cancer Center (P.L.S., M.M., J.C.B.), University of Michigan Medical School, Ann Arbor, Michigan, U.S.A.

Editor's Note: This Manuscript was accepted for publication on November 5, 2018.

J.C.B. received funding from the National Institutes of Health (NIH) grants U01-DE025184, P30-CA046592, R01-CA194536, and ACS132034-RSG-18-062-01-TBG. J.R.B. was supported by NIH grant T32 DC005356-12.

Send correspondence to Chad Brenner, $\mathrm{PhD}, 1150$ E. Medical Center Dr., 9301B MSRB3, Ann Arbor, MI 48109-0602.

E-mail: chadbren@umich.edu

DOI: 10.1002/lary.27725 number of current articles that we believe will influence the future research of liquid biomarkers in HNSCC. As such, our systematic literature search included articles published through September 9, 2018, and was performed using MEDLINE, EMBASE, and Google Scholar for relevant articles. Non-peer-reviewed articles, letters to the editor, commentaries, and editorials were excluded. All review authors independently screened titles and abstracts of potential studies to assess validity (Table I). Subsequently, the full text of all eligible studies was independently reviewed by three review authors (P.L.S., J.R.B., J.с.в.) who evaluated the potential articles for inclusion.

\section{Background to Serum-Based Biomarkers}

As defined by the Biomarkers Definitions Working Group, a biomarker is "a characteristic that is objectively measured and evaluated as an indicator of normal biological processes, pathogenic processes, or pharmacologic responses to a therapeutic intervention." ${ }^{5}$ Biomarkers may offer insight into diseases in many contexts, including screening and diagnosis, yielding predictive information to alter therapy and therapy monitoring or serving as a prognostic tool by risk stratification. All of these roles point to the central role of validating a biomarker with a specific clinical endpoint, ${ }^{5}$ which may be significantly different depending on the point in disease progression (Fig. 1).

The term circulating biomarkers encompasses an array of analytes including proteins, circulating tumor cells (CTCs), ctDNA, and tumor exosomes. These circulating biomarkers have been explored in numerous cancers and situations, including screening, prognostication, evaluating response to therapy, and predicting response to novel therapeutics. Determining prognosis was one of the initial areas in which circulating biomarkers were explored. In nonmetastatic colorectal cancer, for example, 
TABLE I.

Highlighted Liquid Biomarker Studies.

\begin{tabular}{|c|c|c|c|}
\hline Authors & Year & $\begin{array}{c}\text { Number } \\
\text { of Patients }\end{array}$ & Findings \\
\hline Chuang et al & 2008 & 59 & $\begin{array}{l}\text { Quantitative measurement of HPV-16 } \\
\text { DNA in saliva can potentially assist } \\
\text { in surveillance and early detection } \\
\text { of recurrence. Patients with the } \\
\text { presence of HPV- } 16 \text { DNA in } \\
\text { salivary rinses are at significant risk } \\
\text { for recurrence. }\end{array}$ \\
\hline Cheng et al & 2009 & 15 & $\begin{array}{l}\text { ctDNA plasma levels in cancer } \\
\text { patients during radiation therapy } \\
\text { are dynamic throughout the course } \\
\text { of treatment and may provide } \\
\text { real-time assessments of respones } \\
\text { to chemotherapy and radiotherapy }\end{array}$ \\
\hline Ahn et al & 2014 & 93 & $\begin{array}{l}\text { Combined pretreatment plasma and } \\
\text { saliva can increase the utility of } \\
\text { pretreatment HPV-16 as a tool for } \\
\text { screening patients with } \\
\text { HPV-16-positive oropharyngeal } \\
\text { cancer. }\end{array}$ \\
\hline Wang et al & 2015 & 47 & $\begin{array}{l}\text { Sensitivity of HPV-16 DNA detection } \\
\text { varies greatly depending on tumor } \\
\text { location: saliva is more often } \\
\text { enriched for tumor DNA from the } \\
\text { oral cavity, but plasma is more } \\
\text { often enriched for tumor DNA from } \\
\text { the other sites. }\end{array}$ \\
\hline Dahlstrom et al & 2015 & 262 & $\begin{array}{l}\text { Pretreatment serum HPV DNA is } \\
\text { associated with both higher } \mathrm{N} \\
\text { category and overall disease stae } \\
\text { but it does not seem to serve as a } \\
\text { marker for disease recurrence } \\
\text { among patients with oropharyngeal } \\
\text { cancer. }\end{array}$ \\
\hline
\end{tabular}

ctDNA = circulating tumor DNA; HPV = human papillomavirus. both increased baseline ctDNA presurgery and persistently detectable ctDNA postoperatively have been associated with poor prognosis. ${ }^{6,7}$ Enumeration of CTCs in breast cancer has been found to be an independent predictor of survival in metastatic breast cancer. Interestingly, the number of CTCs after 1 month of therapy has been validated to predict survival. Importantly, this is prior to when traditional radiographic imaging response would be expected. ${ }^{8}$ Trials have not demonstrated a benefit to early change in therapy based on CTCs, emphasizing the dual importance of validated tumor biomarkers and effective therapeutics. In addition, research into the use of CTCs in HNSCC have been limited by small cohorts, heterogeneous cancer subtypes, and nonstandardized detection methods. Therefore, this requires further validation and study. ${ }^{9,10}$ Ultimately, comparative work in metastatic breast cancer has suggested that ctDNA is a more sensitive biomarker than enumeration of CTCs, and their dynamics may better reflect tumor response to therapy. ${ }^{11}$

Circulating tumor biomarkers have begun to make a foray into the clinic with the era of precision oncology and targeted therapeutics. This has been most notable in lung adenocarcinoma; studies have demonstrated a ctDNAbased assay to detect targetable mutations and resistance pathways (i.e., EGFR) in the circulation, potentially avoiding biopsy. ${ }^{12-14}$ Genotyping of plasma ctDNA has demonstrated a sensitivity of $74 \%$ to $86.3 \%$ (specificity 96.5\%-100\%) for EGFR exon 19/L858R mutants. Even more compelling is that the mutation most frequently responsible for the loss of EGFR-directed therapy, T790 M, was able to be accurately detected with a

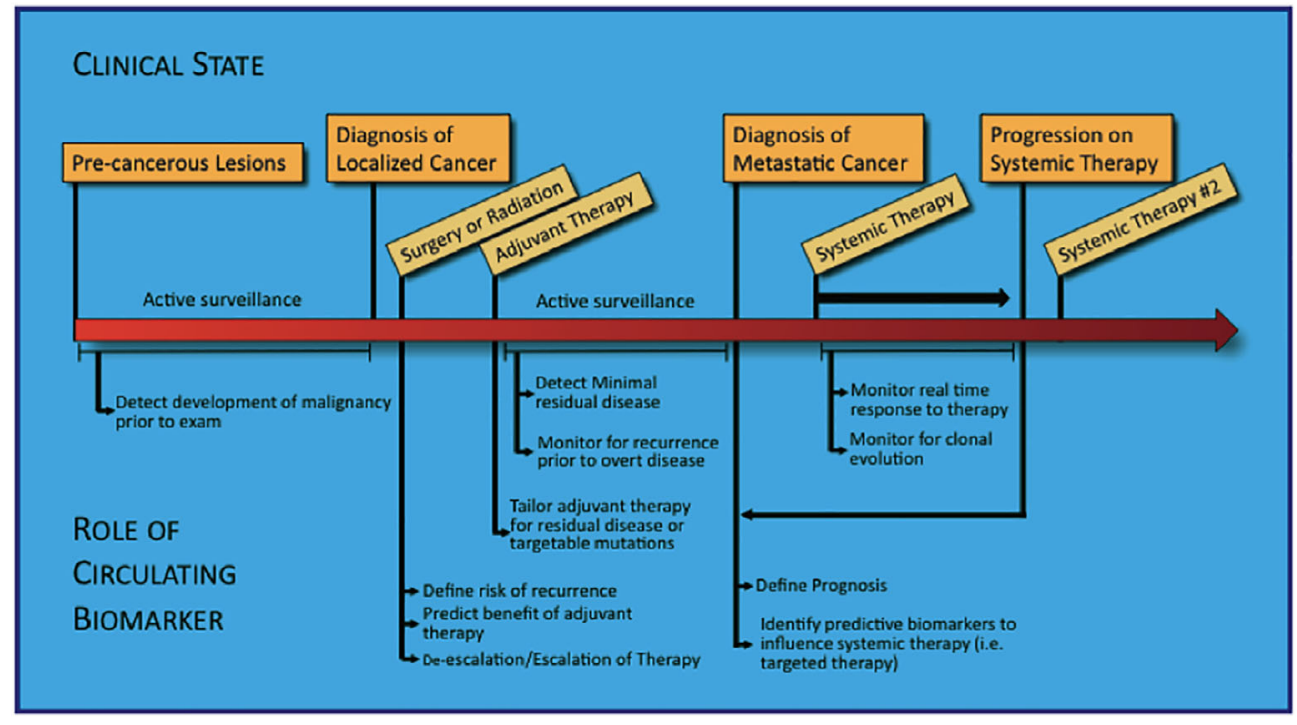

Fig. 1. Circulating biomarkers may have different goals depending on clinical course and intent of the assay. The schematic represents a standardized long-term clinical course for a patient with HNSCC. Treatment modalities are indicated on the top, whereas potential uses of circulating biomarkers are defined on the bottom. In the case of ctDNA assays, endpoints focused on active surveillance of premalignant patients may require a broad ability to detect diverse genetic alterations in order to detect cancers with unknown alterations, whereas active surveillance of patients with a sequenced primary tumor may only require monitoring of a few established alterations (e.g., patient-specific TP53 mutations). Similarly, analysis of genetic changes during clonal outgrowth may also benefit from broad spectrum assays to detect unknown alterations, whereas assays monitoring for treatment-driven selection of therapy-resistant clones (e.g., therapy-driven mutations) may be able to focus on monitoring of highly recurrent mechanisms of resistance for a specific therapeutic. ctDNA = circulating tumor DNA; HNSCC = head and neck squamous cell carcinoma. [Color figure can be viewed in the online issue, which is available at www.laryngoscope.com.] 
sensitivity of $70.3 \%$ to $77 \%$ (specificity $63 \%-100 \%)^{12,13}$ Although this could detect roughly $70 \%$ of EGFR mutations, given the sensitivity, a tumor biopsy may still be warranted in patients with a negative result. Past studies have repeatedly documented that patients with targetable mutations treated with targeted therapies have superior PFS compared to those treated with conventional chemotherapy. ${ }^{15,16}$ Hence, these ctDNA biomarkers are one step closer to achieving the ultimate goal of biomarker development: altering clinical endpoints and improving outcomes. Based on these data, the FDA approved the Cobras EGFR mutation test as first ctDNA biomarker assay for use in clinical practice. Use of similar liquid biopsies is being incorporated into societal guidelines for patients with advanced non-small cell lung cancer. $^{17}$ Each of the circulating tumor biomarkers (e.g., CTCs, ctDNA, exosomes, proteins, antibodies) is distinct with complimentary roles; for this review, we will focus on ctDNA and its potential application in HNSCC.

\section{Introduction to ctDNA}

The concept of ccfDNA (circulating cell-free DNA) correlation with human disease was first introduced in $1948,{ }^{18}$ and increased concentrations of ccfDNA in the circulation of cancer patients was first noted in $1977 .{ }^{19}$ Subsequently, evidence that cfDNA is released into the circulation by tumors (termed ctDNA) was found when tumor-specific aberrations were noted in cfDNA, including tumor suppressor and oncogene mutations, microsatellite instability, and DNA methylation. ${ }^{20-23}$

A variety of biological fluids, including blood, lymph, urine, saliva, and cerebrospinal fluid, contain ccfDNA. In the blood, ccfDNA is predominately short, double-stranded fragments of nuclear and mitochondrial DNA of approximately 160 to 180 base pairs length. ${ }^{24}$ The primary mechanism of DNA release into circulation continues to be somewhat debated; however, the literature suggests that it is a result of apoptosis and possibly also necrosis and viable tumor cell secretion..$^{25,26}$ Lo et al. used whole genome sequencing of plasma DNA of pregnant women to demonstrate that plasma DNA molecules showed a predictable fragmentation pattern consistent with nuclease-cleaved nucleosomes, implicating apoptosis. ${ }^{27}$ Size distribution of cfDNA in healthy individuals and cancer patients also confirms this, revealing an enrichment of size fragments of single or multiples of nucleoprotein complexes, and suggested that the main driver of release may be apoptosis. ${ }^{28}$ Underhill et al. demonstrated that ctDNA fragments may be shorter (approximately 130 base pairs) than ccfDNA derived from noncancer cells. ${ }^{29}$

Through assessment of tumor genetic aberrations in ccfDNA, studies have estimated that the fraction of ctDNA within the total ccfDNA in cancer patients is approximately $10 \% .^{7,30}$ Levels of ccfDNA can be influenced by increased production as well as stability to circulating nucleases and clearance in the kidney, liver, and spleen. Real-time assessment of a cancer may be possible from ctDNA because the half-life of ccfDNA ranges from about 15 minutes to several hours. ${ }^{31-36}$ Some reports indicate that the growth rate of the tumor leads to a higher degree of necrosis, corresponding to an increase in circulating tumor DNA. Diehl et al. suggested that DNA fragments found in the circulation are derived from necrotic neoplastic cells that had been engulfed by macrophages. ${ }^{37}$

\section{Studies to Date of ctDNA in Head and Neck Cancer}

The use of ctDNA in patients with HNSCC is less established than in patients with other cancers such as colorectal, breast, and lung. Nevertheless, research has demonstrated that it is indeed viable, and the potential for applications is vast. ${ }^{32-36}$ The most-studied ctDNA biomarker in HNSCC is HPV-related (HPV+) malignancies because HPV and its downstream protein effectors present as convenient biomarkers for detection in the circulation. $^{38}$ Using real-time polymerase chain reaction (RT-PCR) amplification, a minute amount of HPV-16 DNA has been shown to be detectable in saliva and plasma samples. This study in question included 93 patients with oropharyngeal or unknown primary squamous cell carcinoma with known HPV status and a complete set of pre- and posttreatment plasma or saliva samples. ${ }^{32}$ One study of patients with various stages on HNSCC demonstrated that tumor DNA, as defined by either somatic mutations or HPV incorporation, is detectable in $96 \%$ of patients when both plasma and saliva are sampled. ${ }^{33}$ When considered by body fluid type, the sensitivity of detection varied greatly depending on tumor location. ctDNA was detectable in the saliva samples of between $47 \%$ and $100 \%$ of patients, with the highest detection in tumors of the oral cavity (100\%). Plasma sensitivity was similarly as variable, ranging from $80 \%$ to $100 \%$, highest in the hypopharynx (100\%) (Table II). These complimentary detection rates emphasize the importance of considering which body fluid(s) to evaluate in the development of tests. Interestingly, even when saliva and plasma were combined, tumor DNA was detected in just $86 \%$ of $\mathrm{HPV}+$ patients. $^{33}$

Preliminary research has also suggested that the presence or absence of HPV-16 DNA is a feasible means of surveilling disease posttreatment HPV-16 DNA status in saliva. ${ }^{32,34}$ The detection of HPV-16 DNA in salivary rinses occurred on average over 3 months before clinical detection of recurrence. ${ }^{34}$ In this particularly study, 59 patients were included who presented with histopathologically confirmed HNSCC, had one or more posttreatment salivary sample, and had been previously treated with curative intent. However, only four of these patients had recurrent HPV+ tumors, of which two had detectible HPV-16 in surveillance salivary rinses. A larger study demonstrated that, when combining plasma and salivary surveillance testing, persistence of HPV-16 DNA posttreatment was $69.5 \%$ sensitive and $90 \%$ specific for predicting recurrence within 3 years $^{32}$ (Table II). However, due to the excellent outcomes of HPV-related oropharyngeal cancer, these findings were based on a small number of recurrences. Not all studies have found ctDNA to have clinical utility in predicting disease recurrence in HNSCC. ${ }^{35}$ A study with a different approach assessed the 
TABLE II.

Studies of Circulating Tumor DNA in Head and Neck Cancer.

\begin{tabular}{lccc}
\hline \multicolumn{4}{c}{ ctDNA Detection $\left(\right.$ Wang et al. ${ }^{33}$ ) } \\
\hline Site & $\begin{array}{c}\text { Detected } \\
\text { in Saliva }\end{array}$ & $\begin{array}{c}\text { Detected } \\
\text { in Plasma }\end{array}$ & $\begin{array}{c}\text { Detected in Saliva } \\
\text { or Plasma }\end{array}$ \\
\hline Oral cavity & $100 \%$ & $80 \%$ & $100 \%$ \\
Oropharynx & $47 \%$ & $91 \%$ & $91 \%$ \\
Larynx & $70 \%$ & $86 \%$ & $100 \%$ \\
Hypopharynx & $67 \%$ & $100 \%$ & $100 \%$ \\
HPV+ & $40 \%$ & $86 \%$ & $86 \%$ \\
Total & $76 \%$ & $87 \%$ & $96 \%$
\end{tabular}

HPV-16 DNA as Marker of Recurrence in HPV+ HNSCC

\begin{tabular}{|c|c|c|c|c|}
\hline & $\begin{array}{l}\text { Sample } \\
\text { Source }\end{array}$ & $\begin{array}{c}\text { No. HPV } \\
+ \\
\text { Patients }\end{array}$ & Sensitivity & Specificity \\
\hline $\begin{array}{l}\text { Chuang et al., } \\
2008^{*}\end{array}$ & Saliva & 20 & $50 \%$ & $100 \%$ \\
\hline \multirow[t]{3}{*}{ Ahn et al., $2014^{\dagger}$} & Saliva & 72 & $19 \%$ & $97 \%$ \\
\hline & Plasma & 52 & $55 \%$ & $96 \%$ \\
\hline & $\begin{array}{r}\text { Serum or } \\
\text { plasma }\end{array}$ & 46 & $70 \%$ & $91 \%$ \\
\hline
\end{tabular}

ctDNA detection rates from three of the first HNSCC studies are summarized.

*Testing characteristics for surveillance HPV DNA association with tumor recurrence.

${ }^{\dagger}$ Testing characteristics for posttreatment HPV DNA association with tumor recurrence within 3 years.

ctDNA = circulating tumor DNA; HNSCC = head and neck squamous cell carcinoma; $\mathrm{HPV}+=$ human papillomavirus-related.

use of pretreatment rather than posttreatment ctDNA as a predictor for disease recurrence in oropharyngeal cancer. Pretreatment HPV DNA in the serum was associated with a higher nodal category and overall stage; however, it was not associated with differences in progression-free survival. $^{35}$ This study had a recurrence rate of just $13 \%$ and thus may not have been powered to detect significant associations. Studies to date have lacked the statistical power to validate the role of ctDNA in the detection of recurrence. Confirmation and validation of a candidate biomarker is one of the most challenging issues in translational science. It is a time- and labor-intensive process requiring multiple patient cohorts. Significant literature has been published outlining processes for appropriate clinical biomarker discovery, including independent discovery and validation cohorts prior to clinical evaluation for utility. Clinical evaluation may take one of many designs, including prospective clinical trials in which the test may or may not direct management. Design of a prospective biomarker validation clinical trial requires close collaboration with colleagues in bioinformatics and statisticians, but the sample size is dependent in part on the role of the test. If the biomarker is designed to detect disease recurrence, an ample number of patients treated uniformly must be enrolled to detect an appropriate number of reoccurrences to make appropriate conclusions on the biomarker test characteristics. Even more challenging, in order to define the clinical utility of a proposed biomarker, a study (often as a follow-up) must be designed wherein the results of the biomarker change clinical management. ${ }^{39-41}$ In the situation of HPV-related HNSCC, the recurrence rate is so low that a large cohort is necessary to statistically determine the test characteristics of HPV ctDNA (sensitivity, specificity, area under the curve [AUC]). Furthermore, to solidify its role in clinical practice, a study would need to be designed in which observation would be changed based on detection of HPV ctDNA. For example, if ctDNA was detected, posttreatment patients would be randomized to more intensive surveillance or adjuvant chemotherapy and monitored for improvement in survival. Therefore, although ctDNA may indeed be a potential adjunct to provide less invasive monitoring for recurrent HNSCC, it requires further large prospective studies.

Another application of ctDNA in HNSCC is in assessing real-time responses to chemotherapy and radiotherapy. A study looking at the ctDNA plasma levels in cancer patients during radiation therapy found that concentrations were dynamic throughout the course of treatment. The patients studied had various types of cancer, but more than half of these were localized to the head and neck. In most of the patients, the levels of ctDNA underwent an initial, transient rise at the initiation of therapy, after which they declined. ${ }^{42}$ However, this study was limited both by the variety of malignancies included and by the numerous different treatment modalities. Other studies have demonstrated similar results in patients undergoing radiation and chemotherapy in which there is an initial rise in concentration followed by a slow decline back to pretreatment level. ${ }^{43-46}$ Further research may build upon this foundation to determine whether these dynamic levels of ctDNA correlate with treatment efficacy.

The final application of ctDNA that is currently under investigation in HNSCC is its use in metastatic disease. As aforementioned, research in other malignancies has demonstrated the ability to utilize ctDNA to predict response to chemotherapeutic agents. ${ }^{11}$ It allows for a much less invasive means to select patients who these therapies may benefit. Similarly in lung adenocarcinoma, ctDNA has been able to identify targetable mutations (EGFR, ALK, ROS-1, BRAF) in which targeted therapies are beneficial. ${ }^{14-17}$ Unfortunately, unlike lung adenocarcinoma, to date no driver mutations have been identified in HNSCC, and no mutations have been isolated predictive of response to systemic therapy. Given data regarding the association of tumor mutational burden as assessed by whole exome sequencing in tumor samples and response to immunotherapy, ${ }^{47}$ ongoing research is aiming to define whether this information may be assessed from ctDNA. For example, given the recent emergence of immunotherapies in HNSCC ${ }^{48}$ one recent exploratory study using a commercial next generation sequencing (NGS) ctDNA assay investigated patients with unresectable or metastatic disease who were undergoing checkpoint inhibitor-based immunotherapy. Thirteen percent of the patients in this study had HNSCC. This study suggested that higher alteration number on ctDNA liquid biopsy is associated with a significantly improved response to checkpoint 
A

\begin{tabular}{|l|c|c|c|c|}
\hline \multicolumn{1}{|c|}{ Gene } & $\begin{array}{c}\text { Mutated } \\
\text { Tumors }\end{array}$ & $\begin{array}{c}\text { Percent } \\
\text { Mutated }\end{array}$ & $\begin{array}{c}\text { Gene } \\
\text { Length (nt) }\end{array}$ & Exons* \\
\hline TP53 & 432 & $82 \%$ & 2724 & 12 \\
\hline FAT1 & 123 & $23 \%$ & 14786 & 27 \\
\hline CDKN2A & 114 & $22 \%$ & 1283 & 3 \\
\hline NOTCH1 & 99 & $19 \%$ & 9371 & 34 \\
\hline PIK3CA & 97 & $18 \%$ & 9093 & 21 \\
\hline NSD1 & 72 & $14 \%$ & 12892 & 23 \\
\hline CASP8 & 60 & $11 \%$ & 2930 & 9 \\
\hline EP300 & 38 & $7 \%$ & 9587 & 31 \\
\hline HRAS & 34 & $6 \%$ & 1233 & 6 \\
\hline FBXW7 & 34 & $6 \%$ & 4976 & 12 \\
\hline
\end{tabular}

$\mathrm{B}$

\begin{tabular}{|c|c|c|c|}
\hline $\begin{array}{c}\text { Genomic } \\
\text { Loci }\end{array}$ & $\begin{array}{c}\text { Altered } \\
\text { Tumors }\end{array}$ & $\begin{array}{c}\text { Percent } \\
\text { Altereted }\end{array}$ & $\begin{array}{c}\text { Genes in } \\
\text { locus }\end{array}$ \\
\hline $12 \mathrm{q} 24.33$ & 466 & $88 \%$ & 466 \\
\hline $1 \mathrm{p} 36.21$ & 436 & $82 \%$ & 436 \\
\hline $15 \mathrm{q} 15.1$ & 285 & $54 \%$ & 285 \\
\hline $2 \mathrm{q} 36.2$ & 273 & $52 \%$ & 273 \\
\hline $14 \mathrm{q} 11.2$ & 263 & $50 \%$ & 263 \\
\hline $14 \mathrm{q} 32.32$ & 259 & $49 \%$ & 259 \\
\hline $1 \mathrm{p} 13.2$ & 142 & $27 \%$ & 142 \\
\hline $6 \mathrm{p} 25.3$ & 79 & $15 \%$ & 79 \\
\hline $11 \mathrm{q} 23.1$ & 77 & $15 \%$ & 77 \\
\hline $7 \mathrm{q} 36.1$ & 76 & $14 \%$ & 76 \\
\hline
\end{tabular}

$\mathrm{C}$

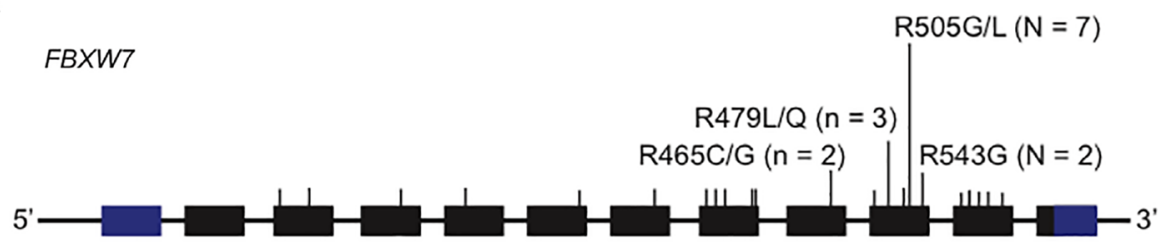

D

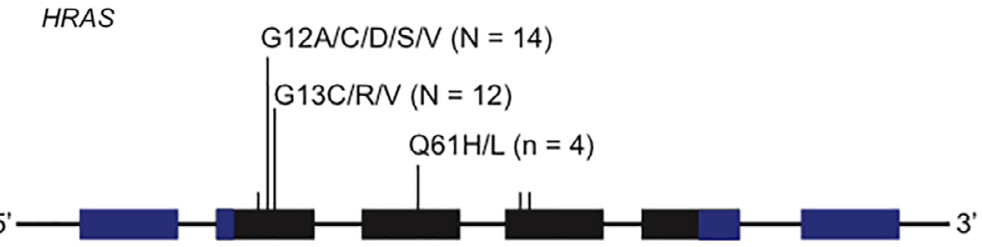

Fig. 2. Challenges in optimizing a ctDNA panel based on the diversity of HNSCC molecular alterations. (A) Top 10 most recurrent molecular alterations in primary untreated disease as defined by the HNSCC TCGA sequencing set $(N=530)$. Several of these genes are tumor suppressors from long, multi-exon genes. (B) Top 10 most recurrent copy number alterations from HNSCC TCGA data and the total genes in each region are indicated. (C) Representation of regions that are required to be covered if ctDNA analysis focuses on tumor suppressors using FBXW7 schematic as an example (all exons would need coverage based on the low recurrence of hot spot alterations). Boxes represent exons, with blue boxes representing untranslated regions and black regions indicating coding sequences. Vertical lines indicate site of called nonsynonymous single nucleotide variant, and only the recurrent mutations were annotated. (D) As in (C), with representative regions of interest in the HRAS oncogene highlighting recurrent mutation sites presented in schematic as shown, suggesting the majority of HRAS mutant patients would be detected with probes covering G12, G13, and Q61. ctDNA = circulating tumor DNA; HNSCC = head and neck squamous cell carcinoma; HPV = human papillomavirus. [Color figure can be viewed in the online issue, which is available at www.laryngoscope.com.]

inhibitor-based immunotherapy. ${ }^{49}$ This is consistent with findings from primary tissue biopsies. Despite the limitations inherent to a study with a smaller HNSCC population, the literature suggests ctDNA may have a role in predicting those metastatic and recurrent patients for whom the prospective therapeutic benefit of immunotherapy is greatest. With further validation, ctDNA could assist in limiting the number of patients who are unnecessarily exposed to the adverse effects of these therapies.

In addition to its use in HPV+ malignancies, biomarkers have also been studied in epstein barr virus (EBV) related nasopharyngeal carcinoma (NPC) as a means to assess treatment response, prognosticate outcome, and screen. Several studies have shown that circulating EBV DNA is detectable in both the plasma and the serum of patients with NPC. ${ }^{50,51}$ In one study, 107 patients with stage IIB-IV NPC had their plasma EBV DNA load tested before, at the midpoint of, and after the culmination of chemoradiation. Of these, there 35 patients failed therapy in whom there was detectable midpoint DNA in 74\%. Detectable midpoint EBV DNA was found to be prognostic of treatment failure and was more predictive of outcomes than was tumor stage. ${ }^{46}$ Another prospective study found that patients with NPC who recurred or metastasized had higher pretreatment plasma or serum EBV DNA concentrations than those who did not. Within the first year after treatment, quantification of plasma EBV DNA was a better adverse prognosticator than disease stage. ${ }^{52}$ Finally, EBV has been studied as a means to screen 20 thousand asymptomatic, ethnically Chinese, middleaged male patients for NPC. Of these, 309 tested persistently positive, and 34 went on to have confirmed NPC; the sensitivity and specificity of the presence of EBV DNA in plasma was found to be $97.1 \%$ and $98.6 \%$, respectively. These patients were detected at earlier stages had better progression-free survival than those in previously studied cohorts. ${ }^{53}$ This study is limited insofar that it had a short follow-up time and thus was unable to evaluate the impact on long-term outcomes, particularly in the setting of the high rate of survival in NPC. ${ }^{54}$ Additionally, the high number needed to screen, and the expenses associated with screening necessitate further evaluation of its cost-effectiveness. Despite these potentially promising applications, the 
clinical utility of EBV DNA as a prognostic biomarker still needs to validated in prospective clinical trials.

\section{Techniques for Analyzing ctDNA}

Recent advancements in single molecule-based NGS technology, coupled with improvements in bioinformatics approaches, have enabled the ability to genotype cancer patients in real time from minimal amounts of ctDNA. In fact, several different techniques have recently been developed to detect and quantify ctDNA in a variety of cancer settings, which can be broadly divided into three main types of assay based on the intent to detect either early stage cancer, changes to molecular pathways driving tumor evolution, and response to therapy or evidence for early recurrence of an already genotyped cancer.

Early detection assays that attempt to discover unidentified cancers are perhaps the most technically challenging because they require highly sensitive methods that assess broad spectrums of the most probable pathogenic alterations. The primary challenge of these assays is the ability to differentiate between somatic events and alterations from either the normal germline or those that occur during hematopoietic cell proliferation, ${ }^{55}$ which are generally thought to require a high depth of sequencing coverage and comprehensive databases of germline and hematopoietic alterations. Due to current limitations in the number of genes that can be cost-effectively sequenced to appropriate depth for high confidence annotation, early detection ctDNA assays tend to rely on panel-based approaches to identify 50 to 100 common genetic alterations found in either an individual cancer or a small set of genetically related cancers. ${ }^{36,56,57}$

For cancers driven by a relatively small set of consensus drivers, early detection assays have the advantage of having to sequence smaller gene sets; in contrast, for cancers such as HNSCC, which are known to have a large and diverse number of oncogenic drivers. ${ }^{58-60}$ The detection of early diagnosis panels becomes slightly more challenging because the number of alterations observed across large multi-exon genes, such as TP53 or NOTCH1, requires significantly more probes and sequencing coverage than oncogenes with "hotspot" molecular alterations occurring at only a few nucleotides in genes, such as HRAS and PIK3CA (Fig. 2).

To overcome these types of challenges, Phallen et al. recently developed a technique called targeted error correction-SEQ, which examines 58 cancer-related genes and uses optimized sequencing and bioinformatics methods to reduce the overall potential of amplification, sequencing, and contamination errors. ${ }^{6}$ Using this method, the authors were about to detect stage 1 or 2 disease in $59 \%$ to $71 \%$ of patients with colorectal, breast, lung, or ovarian cancers representing a large improvement in the ability to detect malignancies. Although this method has not been applied to a large population of patients, it will be interesting to determine the predictive value of the assay for each population.

In contrast to these complex assays to detect early disease or genetic evolution, the assays developed to detect early recurrence have mostly focused on the tracing individual lesions. The rationale for this approach is based on heterogeneity lineage tracing experiments from several different cancer types, suggesting that once a driver lesion occurs in a tumor, it can often be traced in subclones recurring either locally or distantly. ${ }^{61-65}$ In the case of HPV+ HNSCC or mucoepidermoid carcinoma, the development of methods to individually monitor high-risk HPV DNA or CRTC1/3-MAML2 gene fusions, respectively, represents a straightforward approach to develop assays with high specificity for detection. ${ }^{66,67}$ Consequently, once the tumor specific primary lesion has been characterized and likely driver alterations are identified, individualized assays can be developed to trace likely driver lesions. Several examples have emerged in literature that leverage quantitative PCR, RT-PCR, digital droplet PCR, and/or NGS. ${ }^{6-70}$ This method has been especially useful for monitoring recurrence in cancers that are defined by highly recurrent "hotspot" mutations such as BRAF V600E in melanoma; however, even these studies have also shown limits in sensitivity suggesting that monitoring for additional loci may provide an opportunity to improve overall assay performance.

\section{Future Applications of ctDNA in Head and Neck Cancer}

Direct tumor sequencing and mutation detection in plasma cfDNA are unlikely to be a viable clinical approach due to the associated complexity and turnaround time. ${ }^{36}$ However, as previously discussed based on the known mutational landscape of HNSCC, a ctDNA panel could be designed to detect and monitor cases in a rapid and cost-effective fashion. ${ }^{33}$ Use of such a panel would enable researchers and clinicians to detect and monitor non-HPV-related malignancies, which still constitute the majority of HNSCC cases. This would allow trials of previously untreated locally advanced HNSCC to move beyond risk stratification by tumor-node-metastasis stage or HPV status and instead move to real-time response of tumors to therapy as judged by ctDNA dynamics. That could drive intensification of therapy in patients with lack of disease response via ctDNA or deescalatation of therapy based on a fall in ctDNA, thereby preventing treatment-related toxicity. Before either of these designs is pursued, we need not only a validated biomarker test but also an understanding of normal biomarker dynamics during treatment to identify responders/nonresponders.

Development of novel therapeutics in unresectable recurrent or metastatic HNSCC has been met with little success. Cytotoxic chemotherapy can cause severe adverse effects without significant meaningful response, limiting its use and the ability to receive further therapy. Although promising, immunotherapy has been found to have a low response rate of $13 \%$, which is often not appreciated for several months. ${ }^{71-73}$ Novel methods of detecting response are needed to better establish response to therapy and avoid exposure to toxic, ineffective agents. A ctDNA biomarker panel test could detect response prior to traditional radiographic imaging. Analogous to past breast cancer trials, ${ }^{74}$ if a biomarker is able to be validated as predicting response 
prior to conventional imaging (i.e., blood draw after 1 cycle vs. imaging after 3 cycles), a trial evaluating early switch of therapy in those nonresponsive to therapy is quite enticing. Such a trial has the potential to spare patients the toxicities of ineffective therapy and more rapidly administer beneficial therapies. Even more revolutionary would be evaluation of whether clonal resistance can be detected via a ctDNA panel and whether modification of therapy based on clonal resistance would alter patient outcomes. This scenario would take much more development, advancement of bioinformatics, and likely effective targeted therapies.

\section{CONCLUSION}

Although several questions remain, including the cost-effectiveness of in-depth sequencing and improving the speed at which sequencing results are obtained, early studies published thus far have demonstrated promising results for implementing ctDNA monitoring into clinical care. If ctDNA monitoring proves truly impactful, it may not only make routine assessments of cancer progression and response to therapy cheaper and accessible but may also lead to significant improvements in early detection. In addition, we may begin to observe improvements in overall survival for patients with this difficult-to-monitor disease.

\section{BIBLIOGRAPHY}

1. Shield KD, Ferlay J, Jemal A, et al. The global incidence of lip, oral cavity, and pharyngeal cancers by subsite in 2012. CA Cancer $J$ Clin 2017;67: 51-64.

2. Chaturvedi AK, Engels EA, Pfeiffer RM, et al. Human papillomavirus and rising oropharyngeal cancer incidence in the United States. J Clin Oncol 2011;29:4294-4301.

3. Tillman BN, Yanik M, Birkeland AC, et al. Targeted sequencing of an epidemiologically low risk patient defines fibroblast growth factor family aberrations as a putative driver of head and neck squamous cell carcinoma. Head Neck. 2016;38(suppl1):E1646-E1652.

4. Pickering CR, Zhang J, Neskey DM, et al. Squamous cell carcinoma of the oral tongue in young non-smokers is genomically similar to tumors in older smokers. Clin Cancer Res 2014;20:3842-3848.

5. Biomarkers Definitions Working Group. Biomarkers and surrogate endpoints: preferred definitions and conceptual framework. Clin Pharmacol Ther 2001;69:89-95.

6. Phallen J, Sausen M, Adleff V, et al. Direct detection of early-stage cancers using circulating tumor DNA. Sci Transl Med 2017. doi: https://doi.org/10. 1126/scitranslmed.aan2415.

7. Diehl F, Schmidt K, Choti MA, et al. Circulating mutant DNA to assess tumor dynamics. Nature Med 2008;14:985-990.

8. Cristofanilli M, Budd GT, Ellis MJ, et al. Circulating tumor cells, disease progression, and survival in metastatic breast cancer. N Engl J Med 2004; 351:781-791.

9. Guney K, Yoldas B, Ozbilim G, Derin AT, Sarihan S, Balkan E. Detection of micrometastatic tumor cells in head and neck squamous cell carcinoma. A possible predictor of recurrences? Saudi Med J 2007;28:216-220.

10. Kulasinghe A, Perry C, Jovanovic L, Nelson C, Punyadeera C. Circulating tumour cells in metastatic head and neck cancers. Int $J$ Cancer 2015;136: 2515-2523.

11. Dawson SJ, Tsui DW, Murtaza M, et al. Analysis of circulating tumor DNA to monitor metastatic breast cancer. N Engl J Med 2013;368:1199-1209.

12. Sacher AG, Paweletz C, Dahlberg SE, et al. prospective validation of rapid plasma genotyping for the detection of EGFR and KRAS mutations in advanced lung cancer. JAMA Oncol 2016;2:1014-1022.

13. Oxnard GR, Thress KS, Alden RS, et al. Association between plasma genotyping and outcomes of treatment with osimertinib (AZD9291) in advanced non-small-cell lung cancer. J Clin Oncol 2016;34:3375-3382.

14. Wu YL, Sequist LV, Hu CP, et al. EGFR mutation detection in circulating cell-free DNA of lung adenocarcinoma patients: analysis of LUX-Lung 3 and 6. Br J Cancer 2017;116:175-185.

15. Lee CK, Brown C, Gralla RJ, et al. Impact of EGFR inhibitor in non-small cell lung cancer on progression-free and overall survival: a meta-analysis. $J$ Natl Cancer Inst 2013;105:595-605.

16. Mok TS, Wu YL, Ahn MJ, et al. Osimertinib or platinum-pemetrexed in EGFR T790M-positive lung cancer. $N$ Engl J Med 2017;376:629-640.
17. Non-Small Cell Lung Cancer. 2018. Available at: https://www.nccn.org/ professionals/physician gls/pdf/nscl.pdf. Accessed June 16, 2018.

18. Mandel P, Metais $\mathrm{P}$. Les acides nucléiques du plasma sanguin chez l'Homme. Comptes Rendus des Seances de la Societe de Biologie et de ses Filiales 1948;142:241-243.

19. Leon SA, Shapiro B, Sklaroff DM, Yaros MJ. Free DNA in the serum of cancer patients and the effect of therapy. Cancer Res 1977;37:646-650.

20. Wang JY, Hsieh JS, Chang MY, et al. Molecular detection of APC, K- ras, and p53 mutations in the serum of colorectal cancer patients as circulating biomarkers. World J Surg 2004;28:721-726.

21. Stroun M, Anker P, Maurice P, Lyautey J, Lederrey C, Beljanski M. Neoplastic characteristics of the DNA found in the plasma of cancer patients. Oncology 1989;46:318-322.

22. Fujiwara K, Fujimoto N, Tabata M, et al. Identification of epigenetic aberrant promoter methylation in serum DNA is useful for early detection of lung cancer. Clin Cancer Res 2005;11:1219-1225.

23. Shaw JA, Smith BM, Walsh T, et al. Microsatellite alterations plasma DNA of primary breast cancer patients. Clin Cancer Res 2000;6:1119-1124.

24. Thierry AR, Mouliere F, Gongora C, et al. Origin and quantification of circulating DNA in mice with human colorectal cancer xenografts. Nucleic Acids Res 2010;38:6159-6175.

25. Jahr S, Hentze H, Englisch S, et al. DNA fragments in the blood plasma of cancer patients: quantitations and evidence for their origin from apoptotic and necrotic cells. Cancer Res 2001;61:1659-1665.

26. Kidess-Sigal E, Liu HE, Triboulet MM, et al. Enumeration and targeted analysis of KRAS, BRAF and PIK3CA mutations in CTCs captured by a label-free platform: comparison to ctDNA and tissue in metastatic colorectal cancer. Oncotarget 2016;7:85349-85364.

27. Lo YM, Chan KC, Sun H, et al. Maternal plasma DNA sequencing reveals the genome-wide genetic and mutational profile of the fetus. Sci Transl Med 2010;2:61ra91.

28. Heitzer E, Auer M, Hoffmann EM, et al. Establishment of tumor-specific copy number alterations from plasma DNA of patients with cancer. Int $J$ Cancer 2013;133:346-356.

29. Underhill HR, Kitzman JO, Hellwig S, et al. Fragment length of circulating tumor DNA. PLoS Genet 2016;12:e1006162.

30. Newman AM, Bratman SV, To J, et al. An ultrasensitive method for quantitating circulating tumor DNA with broad patient coverage. Nature Med 2014;20:548-554

31. Yu SC, Lee SW, Jiang P, et al. High-resolution profiling of fetal DNA clearance from maternal plasma by massively parallel sequencing. Clinical Chem 2013;59:1228-1237.

32. Ahn SM, Chan JY, Zhang Z, et al. Saliva and plasma quantitative polymerase chain reaction-based detection and surveillance of human papillomavirus-related head and neck cancer. JAMA Otolaryngol Head Neck Surg 2014;140:846-854.

33. Wang Y, Springer S, Mulvey CL, et al. Detection of somatic mutations and HPV in the saliva and plasma of patients with head and neck squamous cell carcinomas. Sci Transl Med 2015;7:293ra104.

34. Chuang AY, Chuang TC, Chang S, et al. Presence of HPV DNA in convalescent salivary rinses is an adverse prognostic marker in head and neck squamous cell carcinoma. Oral Oncol 2008;44:915-919.

35. Dahlstrom KR, Li G, Hussey CS, et al. Circulating human papillomavirus DNA as a marker for disease extent and recurrence among patients with oropharyngeal cancer. Cancer 2015;121:3455-3464.

36. Bettegowda C, Sausen M, Leary RJ, et al. Detection of circulating tumor DNA in early- and late-stage human malignancies. Sci Transl Med 2014; 6:224ra224.

37. Diehl F, Li M, Dressman D, et al. Detection and quantification of mutations in the plasma of patients with colorectal tumors. Proc Natl Acad Sci U S A 2005;102:16368-16373.

38. Sisk EA, Soltys SG, Zhu S, Fisher SG, Carey TE, Bradford CR. Human papillomavirus and p53 mutational status as prognostic factors in head and neck carcinoma. Head Neck 2002;24:841-849.

39. Micheel C, Nass SJ, Omenn GS, et al. Evolution of Translational Omics Lessons Learned and the Path Forward. Washington, DC: National Academies Press; 2012.

40. Hayes DF. Biomarker validation and testing. Molecular Oncol 2015;9: 960-966.

41. McShane LM, Hayes DF. Publication of tumor marker research results: the necessity for complete and transparent reporting. J Clin Oncol 2012;30: $4223-4232$.

42. Cheng C, Omura-Minamisawa M, Kang Y, Hara T, Koike I, Inoue T. Quantification of circulating cell-free DNA in the plasma of cancer patients during radiation therapy. Cancer Sci 2009;100:303-309.

43. Mazurek AM, Rutkowski T, Fiszer-Kierzkowska A, Malusecka E, Skladowski K. Assessment of the total cfDNA and HPV16/18 detection in plasma samples of head and neck squamous cell carcinoma patients. Oral Oncol 2016;54:36-41.

44. Gupta, GP, Kumar S, Marron D, et al. Circulating tumor HPV16 DNA as a biomarker of tumor genomics and disease control in HPV-associated oropharyngeal squamous cell carcinoma. Int J Radiat Oncol Biol Phys 2018; 100:1310-1311.

45. Lo YM, Leung SF, Chan LY, et al. Kinetics of plasma Epstein-Barr virus DNA during radiation therapy for nasopharyngeal carcinoma. Cancer Res 2000;60:2351-2355

46. Leung SF, Chan KC, Ma BB, et al. Plasma Epstein-Barr viral DNA load at midpoint of radiotherapy course predicts outcome in advanced-stage nasopharyngeal carcinoma. Ann Oncol 2014;25:1204-1208. 
47. Rizvi NA, Hellmann MD, Snyder A, et al. Cancer immunology. Mutational landscape determines sensitivity to PD-1 blockade in non-small cell lung cancer. Science 2015;348:124-128.

48. Mann JE, Hoesli R, Michmerhuizen NL, et al. Surveilling the potential for precision medicine-driven PD-1/PD-L1-targeted therapy in HNSCC. $J$ Cancer 2017;8:332-344.

49. Khagi Y, Goodman AM, Daniels GA, et al. Hypermutated circulating tumor DNA: correlation with response to checkpoint inhibitor-based immunotherapy. Clin Cancer Res 2017;23:5729-5736.

50. Mutirangura A, Pornthanakasem W, Theamboonlers A, et al. Epstein-Barr viral DNA in serum of patients with nasopharyngeal carcinoma. Clin Cancer Res 1998;4:665-669.

51. Lo YM, Chan LY, Lo KW, et al. Quantitative analysis of cell-free EpsteinBarr virus DNA in plasma of patients with nasopharyngeal carcinoma. Cancer Res 1999;59:1188-1191.

52. Lo YM, Chan AT, Chan LY, et al. Molecular prognostication of nasopharyngeal carcinoma by quantitative analysis of circulating Epstein-Barr virus DNA. Cancer Res 2000;60:6878-6881.

53. Chan KCA, Woo JKS, King A, et al. Analysis of plasma Epstein-Barr virus DNA to screen for nasopharyngeal cancer. N Engl J Med 2017;377:513-522.

54. Sun X, Su S, Chen C, et al. Long-term outcomes of intensity-modulated radiotherapy for 868 patients with nasopharyngeal carcinoma: an analysis of survival and treatment toxicities. Radiother Oncol 2014;110:398-403.

55. Rube CE, Fricke A, Widmann TA et al. Accumulation of DNA damage in hematopoietic stem and progenitor cells during human aging. PloS One 2011;6:e17487.

56. Olsson E, Winter C, George A, et al. Serial monitoring of circulating tumor DNA in patients with primary breast cancer for detection of occult metastatic disease. EMBO Mol Med 2015;7:1034-1047.

57. Sausen M, Phallen J, Adleff V, et al. Clinical implications of genomic alterations in the tumour and circulation of pancreatic cancer patients. Nat Commun 2015;6:7686.

58. Ludwig ML, Birkeland AC, Hoesli R, Swiecicki P, Spector ME, Brenner JC. Changing the paradigm: the potential for targeted therapy in laryngeal squamous cell carcinoma. Cancer Biol Med 2016;13:87-100.

59. Michmerhuizen NL, Birkeland AC, Bradford CR, Brenner JC. Genetic determinants in head and neck squamous cell carcinoma and their influence on global personalized medicine. Genes Cancer 2016;7:182-200.

60. Giefing M, Wierzbicka M, Szyfter K, et al. Moving towards personalised therapy in head and neck squamous cell carcinoma through analysis of next generation sequencing data. Eur $J$ Cancer 2016;55:147-157.

61. Warrick JI, Hovelson DH, Amin A, et al. Tumor evolution and progression in multifocal and paired non-invasive/invasive urothelial carcinoma. Virchows Arch 2015;466:297-311.
62. Sidransky D, Frost P, Von Eschenbach A, Oyasu R, Preisinger AC Vogelstein B. Clonal origin of bladder cancer. N Engl J Med 1992;326: $737-740$

63. Sidransky D, Mikkelsen T, Schwechheimer K, Rosenblum ML, Cavanee W, Vogelstein B. Clonal expansion of p53 mutant cells is associated with brain tumour progression. Nature 1992;355:846-847.

64. Furusato B, Tan SH, Young D, et al. ERG oncoprotein expression in prostate cancer: clonal progression of ERG-positive tumor cells and potential for ERG-based stratification. Prostate Cancer Prostatic Dis 2010;13 228-237.

65. Shi H, Hugo W, Kong X, et al. Acquired resistance and clonal evolution in melanoma during BRAF inhibitor therapy. Cancer Discov 2014;4: 80-93.

66. Birkeland AC, Foltin SK, Michmerhuizen NL, et al. Correlation of Crtc1/3Maml2 fusion status, grade and survival in mucoepidermoid carcinoma. Oral Oncol 2017;68:5-8.

67. Capone RB, Pai SI, Koch WM, et al. Detection and quantitation of human papillomavirus (HPV) DNA in the sera of patients with HPV-associated head and neck squamous cell carcinoma. Clin Cancer Res 2000;6: 4171-4175.

68. Thress KS, Brant R, Carr TH, et al. EGFR mutation detection in ctDNA from NSCLC patient plasma: A cross-platform comparison of leading technologies to support the clinical development of AZD9291. Lung Cancer 2015;90:509-515.

69. Tsao SC, Weiss J, Hudson C, et al. Monitoring response to therapy in melanoma by quantifying circulating tumour DNA with droplet digital PCR for BRAF and NRAS mutations. Sci Rep 2015;5:11198.

70. Reinert T, Scholer LV, Thomsen R, et al. Analysis of circulating tumour DNA to monitor disease burden following colorectal cancer surgery. Gut 2016;65:625-634.

71. Bauml J, Seiwert TY, Pfister DG, et al. Pembrolizumab for platinum- and cetuximab-refractory head and neck cancer: results from a single-arm, phase II study. J Clin Oncol 2017;35:1542-1549.

72. Ferris RL, Blumenschein G, Fayette J, et al. Nivolumab for Recurrent squamous-cell carcinoma of the head and neck. N Engl J Med 2016;375 1856-1867.

73. Chow LQM, Haddad R, Gupta S, et al. Antitumor activity of pembrolizumab in biomarker-unselected patients with recurrent and/or metastatic head and neck squamous cell carcinoma: results from the phase Ib KEYNOTE-012 expansion cohort. J Clin Oncol 2016;34: $3838-3845$.

74. Smerage JB, Barlow WE, Hortobagyi GN, et al. Circulating tumor cells and response to chemotherapy in metastatic breast cancer: SWOG S0500. J Clin Oncol 2014;32:3483-3489. 